\title{
Motivação e desempenho tático em jovens jogadores de futebol: uma análise a partir da teoria da autodeterminação
}

\author{
Motivation and tactical performance in young soccer players: an \\ analysis from the theory of self-determination
}

Paulo Henrique Borges', Deisy de Oliveira Silva', Edielson Frigeri Leite Ciqueira', leda Parra Barbosa Rinaldi’, Wilson Rinaldi', Lenamar Fiorese Vieira'

'Universidade Estadual de Maringá, Maringá, PR, Brasil.

Recebido em: junho 2015 / Aceito em: agosto 2015

paulo.borges.proesporte@gmail.com

\section{RESUMO}

O entendimento dos fatores motivacionais que interferem no desempenho tático dos jogadores de futebol é fundamental para auxiliar as intervenções dos treinadores, durante o processo de treinamento. Objetivo: analisar as relações dos níveis motivacionais com o desempenho tático de jogadores de futebol. Método: fizeram parte da amostra 29 jogadores de futebol $(15,38$ anos $\pm 1,20$ ), pertencentes a um projeto de extensão da Universidade Estadual de Maringá. Para avaliar a motivação, foi utilizada a Escala de Motivação para o Esporte (SMS); para avaliar o desempenho tático, foi utilizado o Game Performance Assessment Instrument (GPAI). Para analisar os dados, foi utilizado o teste U-Mann Whitney e o Coeficiente de Correlação de Spearman. Resultados: os jogadores juvenis apresentaram maior desmotivação em relação aos infantis $(p=0,03)$. Foram identificadas correlações moderadas entre execução de habilidade $(r=0,53)$ e desempenho tático $(r=0,43)$ com a motivação intrínseca. Considerações finais: os jogadores com maior nível de motivação intrínseca apresentam melhor desempenho tático e execução de habilidades específicas da modalidade. Assim, sugere-se que a motivação intrínseca é um fator que pode contribuir para a manifestação das qualidades técnicas e táticas em jovens jogadores de futebol.

Palavras-chave: Avaliação; Tática; Motivação; Jovens.

\section{ABSTRACT}

Understanding the motivational factors that interfere with the tactical performance of soccer players is crucial to support the activities of the coaches during the training process. Objective: analyze and correlate the motivational levels with the tactical performance of young soccer players. Method: the sample consisted of 29 soccer players (15.38 years \pm 1.20 ) belonging to an extension project at the State University of Maringá. To assess motivation was used Scale Motivation for Sport (SMS). To assess the tactical performance, we used the Game Performance Assessment Instrument (GPAl). To investigate the differences between the categories and correlate variables, the Mann-Whitney $U$ test and the Spearman correlation coefficient was used. Results: that juvenile players had higher motivation towards children $(p=0,03)$ and moderate correlation between running ability $(r=0,53)$ and tactical performance $(r=0,43)$ with intrinsic motivation. Closing remarks: that players with higher level of intrinsic motivation have better performance and tactical execution of specific skills of the sport. Thus, it is suggested that intrinsic motivation is a factor that can contribute to the manifestation of technical and tactical qualities in young soccer players.

Keywords: Evaluation; Tactics; Motivation; Young.

\section{INTRODUÇÃO}

A avaliação do desempenho tático de jovens jogadores é de suma importância para o processo de ensino do esporte, sobretudo no que diz respeito ao treinamento das categorias de base, onde o processo de ensino-aprendizagem se dá de forma mais intensa e também onde ocorrem as seleções dos talentos esportivos. ${ }^{1}$ 
Treinadores e técnicos, de diferentes modalidades esportivas, buscam por instrumentos que possam auxiliar nos procedimentos de avaliação e que sejam, ao mesmo tempo, aplicáveis de acordo com os objetivos e fases de periodização dos treinos e das competições. Tal realidade se dá também no Futebol e, destarte, a avaliação tática, nesse esporte, tem sido objeto de estudo de muitas pesquisas na busca pela construção de instrumentos capazes de avaliar de forma adequada o desempenho em jogo dos atletas e seus desdobramentos. ${ }^{2}$

Costa $^{3}$ aponta que os instrumentos para avaliar o comportamento tático se distinguem com base em dois tipos de conhecimento avaliado: o conhecimento declarativo e o processual. O conhecimento declarativo trata-se da revelação verbal e/ou escrita do jogador sobre a melhor decisão a ser tomada em situações específicas de jogo ou treino, enquanto no conhecimento processual o jogador operacionaliza, de forma prática (motora), respostas julgadas apropriadas aos problemas ocorridos das situações de jogo e treino.

$\mathrm{Na}$ avaliação da componente tática, outros fatores estão relacionados ao desempenho em jogo dos atletas, como as componentes técnicas, físicas e psicológicas. Pesquisas, da área da Psicologia Esportiva, investigam os fatores psicológicos determinantes e que influenciam a prática esportiva e/ou de exercícios físicos, dentre os quais a motivação aparece de forma significativa, caracterizada como um processo ativo, intencional e dirigido a uma meta, dependente da interação de fatores pessoais (intrínsecos) e ambientais(extrínsecos).

Atualmente, a teoria que melhor aborda a motivação intrínseca e extrínseca é a Teoria da Autodeterminação (TAD), de Deci e Ryan, ${ }^{4}$ que tem como base, a relação dialética entre o organismo vivo e o contexto social e, diferentemente de algumas percepções anteriores que tomavam a motivação extrínseca e intrínseca como constructos independentes. Essa abordagem propõe a motivação como um continuum caracterizado por níveis de autodeterminação, desde o menos autodeterminado (desmotivação e motivação extrínseca) ao mais autodeterminado (motivação intrínseca).

Pesquisas assinalam que em níveis mais elevados de motivação os atletas tendem a melhorar o seu desempenho, frente a sentimentos de desafio, prazer e autoconfiança, direcoionam suas energias em prol dos seus objetivos e também dos objetivos da equipe em esforço máximo para se alcançar o melhor resultado. ${ }^{5-10}$

Entretanto, apesar da relevância e dos inúmeros estudos que abordam a motivação e o desempenho tático, percebe-se que há uma carência na literatura em relação a pesquisas que relacionem estas temáticas no tocante ao treinamento de adolescentes no futebol.

Nesse sentido, este estudo tem como objetivo analisar as relações entre os níveis motivacionais e de desempenho tático de jogadores de futebol de campo. Com base no suporte teórico, acredita-se que os resultados apontem relações significativas entre a motivação intrínseca e o ótimo desempenho em jogo, enquanto níveis de motivação extrínseca e a desmotivação assinalem baixos níveis de desempenho.

\section{MÉTODO}

Este estudo foi aprovado pelo Comitê de ética local (parecer $n^{\circ}$ 653.698). Trata-se de uma pesquisa transversal de caráter descritivo exploratório, com a intenção de proporcionar maior familiaridade com a problemática em questão, realizando descrições precisas acerca dos fatos em busca de propiciar uma nova visão sobre o mesmos. ${ }^{11-14}$

Foram pré-selecionados a participar do estudo 55 jogadores com idade entre 14 e 17 anos, agrupados em duas categorias de jogo infantil $(n=11)$ e juvenil $(n=18)$, pertencentes ao projeto de extensão da Universidade Estadual de Maringá denominado Centro de Formação em Futebol (Proc. 8849/2010), estabelecido no Departamento de Educação Física (DEF). Adotaram-se os seguintes critérios de inclusão: (1) participar de treinamento sistematizado na modalidade por pelo menos dois anos; (2) ter assiduidade nos treinamentos semanais relatada pelo treinador ( $3 x$ por semana); (3) participar de competições regionais e (4) assinar o Termo de Consentimento Livre e Esclarecido (TCLE). Dessa forma, a amostra final foi constituída por 29 jogadores de futebol $(15,38$ anos $\pm 1,20)$.

Para a coleta de dados, foi realizado um contato inicial com o coordenador do Centro de Formação em Futebol, o qual autorizou a realização da pesquisa. Após esta etapa, foram entregues aos jogadores o TCLE. Todos os que atenderam aos critérios de inclusão foram convidados a comparecer no campo de futebol para a realização dos testes.

Para avaliar o nível motivacional dos jogadores utilizou-se a Escala de Motivação para o Esporte (SMS - Sport Motivation Scale), proposto por Brière e colaboradore ${ }^{15}$ e validada para a lingua portuguesa por Barra e colaboradores. ${ }^{16}$ A escala consiste em 28 questões divididas em sete subescalas avaliadas em uma escala Likert de sete pontos. A Escala de Motivação para o Esporte avalia a motivação de acordo com pressupostos da Teoria da Autodeterminação de Ryan e Deci. ${ }^{4}$ Divide-se a escala em desmotivação (sentimento de desesperança e incompetência, falta de interesse, de controle e vontade em realizar determinada tarefa), motivação extrínseca de motivação externa (comportamento motivado por ameaças, recompensas e pressão familiar), motivação extrínseca de introjeção (pressões internas para realizar uma tarefa), motivação extrínseca de identificação (comportamento percebido como pessoalmente importante e útil, tal como praticar esporte para melhorar a saúde), motivação intrínseca para atingir objetivos (relacionados a fatores em que o atleta sente prazer na busca de objetivos e habilidades esportivas), motivação intrínseca para experiências estimulantes (busca de experiências no esporte que possam proporcionar prazer e diversão) e motivação intrínseca para conhecer (ligada a curiosidade e busca para compreender os fatores esportivos por desejo próprio).

Para melhor tratamento dos dados, as subcategorias "motivação externa, introjeção e identificação" foram agrupadas e formaram a categoria motivação extrínseca. Já, as subcategorias “motivação para atingir objetivos, para experiências estimulantes e para conhecer" foram agrupadas e representadas apenas por motivação intrínseca. A categoria desmotivação também foi contabilizada. $\mathrm{O}$ valor do alfa de Cronbach para todas as dimensões foi considerado forte $(\alpha=0,81)$. 
O desempenho tático foi avaliado por meio do Game Performance Assessment Instrument (GPAI) proposto por Oslin e colaboradores. ${ }^{17}$ Este instrumento foi desenvolvido para mensurar o desempenho em jogo e os comportamentos que demonstram entendimento tático na modalidade, assim como a capacidade dos jogadores resolverem os problemas do jogo utilizando habilidades adequadas. Embora o GPAI possua sete componentes de análise, optou-se, neste estudo, pelo uso de três componentes do jogo para estimar o desempenho dos jogadores, tal como sugerem Oslin e colaboradores ${ }^{17}$ para análise do jogador de futebol: tomada de decisão (subdividido em apropriada (A) e inapropriada (I) de acordo com a situação do jogo), execução de habilidade (subdividido em Eficaz (E) e Ineficaz (I), de acordo com sua execução) e apoio (subdividido em apropriada e inapropriada).

A tomada de decisão refere-se a decisões que o jogador escolhe ao longo do jogo que sejam benéficas para a sua equipe mediante o contexto, como por exemplo, fazer um passe a um companheiro desmarcado. A execução de habilidade diz respeito à utilização de técnicas adequadas para jogar o jogo, como chutar, passar e dominar com qualidade. Já, o apoio é toda ação realizada para desmarcar-se, visando receber a bola, mostrar-se para o companheiro de equipe que possui a bola, criando uma linha de passe.

Durante a avaliação, cada jogador começa com zero pontos e ganha um ponto por tomada de decisão apropriada/Execução de habilidade eficaz/apoio apropriada e um ponto por tomada de decisão inapropriada (I)/Execução de habilidade ineficaz/apoio inapropriado. Após este procedimento, é possível chegar a três índices de acordo com Memmert e colaboradores: ${ }^{18}$ Índice de Tomada de Decisão, ITD $=[A /(A+I)]$, Índice de Execução de habilidade, $I E H=[A /(A+I)]$ e Índice de Apoio, $I A=[A / A+I)]$. Portanto, o desempenho no jogo (DJ) de cada jogador varia de 0 a 1 e pode ser conseguido da seguinte maneira, $D J=I T D+I E H+I A / 3$. Sendo assim, cada jogador foi avaliado por três professores de Educação Física. Após as avaliações, foi realizada a média dos valores de cada índice.

\section{Procedimentos}

Os jogadores, primeiramente responderam à Escala de Motivação para o Esporte (SMS). Posteriormente, foram para o campo de futebol e foram orientados a realizar um aquecimento prévio, em torno de 10 minutos. Após o aquecimento, os jogadores foram divididos pelo treinador em equipes de três jogadores, agrupados de acordo com o seu nível de jogo.

Os jogos tiveram duração de 5 minutos e foram filmados por uma filmadora digital Canon EOS Rebel 18.0 megapixels de resolução, colocada a $2 \mathrm{~m}$ do chão e posicionada em diagonal, em relação às linhas de fundo e lateral, capaz de filmar toda a extensão da área de jogo. A estrutura de cada equipe foi $3 \times 3$ (goleiro +3 jogadores vs goleiro +3 jogadores), uma vez que conforme relatos de Garganta ${ }^{19}$ essa estrutura revela-se como sendo a configuração mínima que garante a ocorrência de todos os princípios táticos inerentes ao jogo formal. Os jogadores foram enumerados de 1 a 6 (1 a 3 de uma cor e 4 a 6 de outra cor), cujo objetivo era facilitar a identificação dos jogadores no vídeo. A dimensão do campo utilizada foi $36 \mathrm{~m} \times 27 \mathrm{~m}$.

\section{Análise estatística}

Foi utilizado o pacote estatístico SPSS 20.0. Para verificar a normalidade dos dados, utilizou-se o teste Shapiro-Wilk. Identificada a necessidade de se utilizar estatística não paramétrica, aplicou-se o teste U-Mann Whitney para comparação dos grupos sub-15 e sub-17 entre as subescalas motivacionais. Já, para a correlação das variáveis de desempenho em jogo e de motivação, utilizou-se o Coeficiente de Correlação de Spearman. Após verificar a esfericidade dos dados, foi utilizado o teste Friedman para verificar se houve diferença entre as subescalas de motivação, seguido pelas comparações aos pares do teste Wilcoxon. Para avaliar a fidedignidade inter-avaliadores ( $A, B$ e $C$ ), foi utilizado o Índice Kappa. Os valores obtidos entre $A B(0,93), A C(0,94)$ e $B C(0,87)$ revelaram boa concordância inter-avaliadores. $O$ valor de significância adotado foi $p \leq 0,05$.

\section{RESULTADOS}

Os dados da Tabela 1 apontam as comparações das medianas e do primeiro e terceiro quartis das subescalas de motivação dos jogadores de futebol de campo de ambas categorias, sub-15 e sub-17.

Tabela 1 - Comparação entre as subescalas de motivação dos jogadores de futebol de campo das categorias infantil e juvenil.

\begin{tabular}{lcc}
\hline Subescala de motivação & Md & (01-03) \\
\hline Motivação intrínseca & $5,66 \mathrm{a} / \mathrm{b}$ & $(5,00-6,33)$ \\
Motivação extrínseca & $4,75^{\mathrm{a}} / \mathrm{c}$ & $(4,29-5,41)$ \\
Desmotivação & $2,75 \mathrm{~b} / \mathrm{c}$ & $(1,62-3,75)$ \\
\hline
\end{tabular}

a: Diferença significativa entre motivação intrínseca e extrínseca; b: Diferença significativa entre motivação intrínseca e desmotivação; c: Diferença significativa entre motivação extrínseca e desmotivação. *Diferença significativa $p<0,05$.

Nota-se que a subescala de motivação intrínseca foi a mais evidenciada entre os jogadores de futebol $(p=0,001)$, uma vez que apresentou maiores medianas. Já, a desmotivação foi a dimensão com menores índices, apresentando diferença significativa com as subescalas de motivação intrínseca e extrínseca.

A seguir, a Tabela 2 apresenta as comparações das medianas e do primeiro e terceiro quartis relativas às subescalas de motivação de jogadores de futebol de campo, das categorias infantil (sub-15) e juvenil (sub17). Os valores da subescala desmotivação apresentou diferença significativa entre as categorias $(p=0,03)$, revelando que a categoria sub-17 ( $\mathrm{Md}=2,87)$ apresenta maior nível de desmotivação em relação à categoria sub-15 (Md=1,75).

Tabela 2 - Comparação entre as variáveis motivacionais entre os grupos sub-15 e sub-17.

\begin{tabular}{lccc}
\hline Dimensões & $\begin{array}{c}\text { Sub-15 (n=11) } \\
\text { Md (01-03) }\end{array}$ & $\begin{array}{c}\text { Sub-17 }(\mathbf{n}=18) \\
\text { Md (01-03) }\end{array}$ & $\mathbf{~ p ~}$ \\
\hline Motivação Intrinseca & $5,66(5,33-6,16)$ & $5,54(4,91-6,45)$ & 0,88 \\
Motivação Extrinseca & $4,75(4,00-5,33)$ & $4,83(4,31-5,41)$ & 0,87 \\
Desmotivação & $1,75(1,25-3,25)$ & $2,87(2,18-4,00)$ & $0,03 *$ \\
\hline${ }^{*}$ Diferenca significativa, adotando $p \leq 0,05$ & &
\end{tabular}

*Diferença significativa, adotando $p \leq 0,05$. 
Tabela 3 - Correlação entre as variáveis motivacionais e de desempenho tático em jogadores de futebol das categorias sub-15 e sub-17.

\begin{tabular}{lccc}
\hline & Motivação Intrínseca & Motivação Extrinseca & Desmotivação \\
$\mathbf{r}(\mathbf{p})$ & $0,28(0,14)$ & $-0,02(0,91)$ & $0,18(0,34)$ \\
\hline Tomada de decisão & $0,53(0,03)^{*}$ & $0,32(0,08)$ & $-0,16(0,39)$ \\
Execução de Habilidade & $0,04(0,83)$ & $0,15(0,44)$ & $-0,08(0,68)$ \\
Apoio & $0,43(0,02)^{*}$ & $0,24(0,21)$ & $-0,02(0,90)$ \\
Desempenho tático & & &
\end{tabular}

*Correlação significativa, adotando $p \leq 0,05$.

Percebe-se, ao correlacionar as variáveis motivacionais e de desempenho em jogo, correlações significativas entre os componentes execução de habilidade e desempenho em jogo com a motivação intrínseca ( $r=0,53$ e $r=0,43$, respectivamente), como assinala a Tabela 3 .

\section{DISCUSSÃO}

Este estudo teve por objetivo analisar as relações entre os níveis motivacionais e o desempenho tático de jogadores de futebol de campo, embasando-se na Teoria da Autodeterminação. ${ }^{4}$ Apesar das pesquisas publicadas sobre a TAD, não foram encontrados estudos que relacionassem o desempenho em jogo, com a teoria em questão.

Observou-se que os jogadores de ambas as categorias pesquisadas se mostraram autodeterminados para a prática do futebol. Tal constatação fica evidente na tabela 1, ao analisarmos os valores elevados de motivação intrínseca $(M d=5,66)$, sobressaindo-se, tanto em relação à motivação extrínseca quanto à desmotivação que, por sua vez, foi a menos evidenciada ( $M d=2,75)$. Tais resultados sugerem que os jogadores sentem prazer pela prática do futebol e gostam de fazê-lo, corroborando com as achados de Vieira e colaboradores ${ }^{20}$ que, num estudo acerca da relação entre os estilos de suporte parental e a motivação de atletas de futebol de campo de categorias infantil e juvenil, também apontou menor aparição da desmotivação e maior evidência da motivação intrínseca nesta população, bem como com o estudo de Garcia-Mas, ${ }^{21}$ no qual foram identificados os mesmos resultados. Isso demonstra que a motivação dos jogadores das categorias pesquisadas tem origem em fatores internos ao executar a tarefa, envolvendo sentimentos como a satisfação e o divertimento inerente à modalidade.

Já, no que diz respeito à desmotivação, não se podem fazer as mesmas relações com tais autores supra citados. Enquanto no estudo de Vieira ${ }^{20}$ não foram encontradas diferenças significativas nas variáveis motivacionais entre as categorias infantil e juvenil e, em Garcia-Mas, ${ }^{15}$ a motivação dos jogadores da categoria juvenil foi identificada como intrínseca, nossos resultados apontam outra realidade, quando comparadas às categorias sub-15 e sub-17, haja vista que verificou-se que a categoria juvenil se apresentou mais desmotivada em relação à categoria infantil, apontando diferença estatisticamente significativa $(p \leq 0,05)$.

Os resultados encontrados também contradizem aos do estudo de Balbinotti e colaboradores, ${ }^{22}$ no qual buscaram descrever o comportamento do fator Prazer para a atividade física em adolescentes do sexo masculino com idades entre 13 e 19 anos, em que verifi- cou que estes estavam altamente (e intrinsecamente) motivados, durante toda a fase da adolescência, não havendo diferença significativa entre as idades.

No mais, não foram encontradas pesquisas que venham ao encontro dos achados no presente estudo sobre a desmotivação entre as categorias. Estima-se que tais resultados sobre a desmotivação dos jogadores da categoria juvenil possam estar relacionados a fatores como a diminuição e/ou inexistência de maiores expectativas de avançar como atleta na modalidade, uma vez que até então não houve grandes avanços e convites de clubes, enquanto os jogadores da categoria infantil ainda têm um caminho maior a percorrer e a esperança e chances de evolução ainda se fazem maiores.

Foi demonstrada correlação significativa entre a motivação intrínseca com a execução de habilidade $(r=0,53)$ e o desempenho tático $(r=0,43)$ dos jogadores, indicando que os atletas com maior interesse e prazer pela tarefa apresentam melhores índices, tanto ao executar habilidades específicas da modalidade como chutar, passar e dominar, quanto com o desempenho tático global na modalidade, demonstrando melhor resolução dos problemas frente ao contexto tático que o jogo apresenta (Tabela 3). As variáveis Tomada de decisão e Apoio não demonstraram correlações significativas. Contudo, num estudo realizado com jogadores de futebol com idades entre 12 e 16 anos na Espanha por Álvarez e colaboradores, ${ }^{23}$ verificou-se que jogadores adolescentes com maior motivação autodeterminada possuem melhor engajamento e relação interpessoal bem como se sentem mais competentes em seu esporte e mais autônomos em suas ações, uma vez que estas características estão diretamente ligadas ao apoio e a atmosfera criada por figuras de autoridade - como os treinadores - e à satisfação de suas necessidades psicológicas básicas. Outros autores indicam ainda que níveis elevados de motivação intrínseca contribuem para a melhora do desempenho, uma vez que os jogadores canalizam suas energias em prol de seus objetivos, buscando atingir resultados cada vez melhores..$^{5-7}$

\section{CONSIDERAÇÕES FINAIS}

A motivação intrínseca se mostrou significativamente correlacionada ao desempenho tático dos jogadores, confirmando as hipóteses iniciais do estudo de que sujeitos intrinsecamente motivados têm melhor desempenho em jogo, do que os extrinsecamente motivados ou desmotivados. Os resultados obtidos confirmam os pressupostos da Teoria da Autodeterminação, sugerindo que pessoas mais autodeterminadas tem maiores probabilidades de engajamento em deter- 
minados comportamentos e/ou práticas do que pessoas com baixa autodeterminação e, portanto, confirmam a legitimidade da utilização da TAD, em estudos sobre o desempenho tático de atletas.

Esta pesquisa aponta algumas limitações tais como a amostra restrita a apenas um local de treinamento e também pertencentes a um único município, decorrentes do tipo de estudo e também de parte dos instrumentos utilizados, os quais dificultam a realização em grandes escalas num curto período de tempo.

Os resultados encontrados e discutidos nesse trabalho aparecem como subsídio teórico para que os envolvidos na preparação de jovens jogadores de futebol possam levar em consideração as implicações e a importância da motivação no desempenho tático e na resolução de tarefas motoras.

Sugere-se ainda, a partir deste estudo, que futuras pesquisas avancem em relação à esta temática e investiguem mais afundo as questões, causas e fatores relacionados à motivação e ao desempenho em jogo de atletas de futebol de campo nas diferentes categorias e apontem suas implicações para o contexto esportivo.

\section{REFERÊNCIAS}

1. Scaglia AJ, Reverdito RS, Leonardo L, Lizana CJR. O ensino dos jogos esportivos coletivos: as competências essenciais e a lógica do jogo em meio ao processo operacional sistêmico. Movimento (Porto Alegre) 2013; 19(4): 227-249.

2. Tenga A, Kanstad D, Ronglan LT, Bahr R. Developing a New Method for Team Match Performance Analysis in Professional Soccer and Testing its Reliability. Int. J. of Perf. Anal. Sport 2009; 9(1): 8-25.

3. Costa IT. Comportamento Tático no Futebol: Contributo para a avaliação do Desempenho de Jogadores em situações de Jogo Reduzido. Porto: I. T. Costa. Tese (Doutorado em Ciências do Desporto) - Faculdade de Desporto da Universidade do Porto, Porto, 2010.

4. Deci EL, Ryan RM. Intrinsic motivation and self-determination in human behavior. New York: Plenum, 1985.

5. Gould D, Guinan D, Greenleaf C, Medbery R, Peterson K. Factors affecting olympic performance: Perceptions of athletes and coaches from more and less successful teams. The Sports Psychology 1999; 13(1): 371-394.

6. Corrêa DKA, Alchieri JC, Duarte LRS, Strey MN. Excelência na produtividade: A Performance dos Jogadores de Futebol Profissional. Psicologia: Reflexão e crítica 2002; 15(2): 447-460. DOI: http://dx.doi.org/10.1590/S0102-79722002000200021.

7. Hallal PC, Nascimento RR, Hackbart L, Rombaldi AJ. Fatores Intervenientes Associados ao Abandono do Futsal em Adolescentes, R. bras. Ci. e Mov 2004; 12(3): 27-32.

8. Vissoci, JRN, Vieira LF, Oliveira LP, Vieira JL. Motivação e atributos morais no esporte. Revista da Educação Física/ UEM Maringá 2008; 19(2): 173-182. DOI: http://dx.doi. org/10.4025/reveducfisv19n2p173-182.

9. Massa M, Uezu R, Böhme MTS. Judocas olímpicos brasileiros: fatores de apoio psicossocial para o desenvolvimento do talento esportivo. Rev Bras Educ Fís Esporte, São Paulo 2010; 24(4): 471-81. DOI: http://dx.doi.org/10.1590/S180755092010000400005.

10. Ferreira RM, Penna EM, Costa VT, Moraes LCCA. Nadadores medalhistas olímpicos: contexto do desenvolvimento brasileiro. Motriz, Rio Claro 2012; 18(1): 130-142.

11. Cervo AL, Bervian PA, Silva R. Metodologia científica. 6. ed. São Paulo: Pearson Prentice Hall, 2007.

12. Lakatos, EM, Marconi MA. Fundamentos de metodologia científica. 4. ed. rev. e ampl. São Paulo: Atlas, 2001.

13. Hair Jr JF, Babin B, Money AH, Samouel P. Fundamentos de métodos de pesquisa em Administração. Porto Alegre: Bookman, 2005.

14. Gil, AC. Como elaborar projetos de pesquisa. 4. ed. São Paulo: Atlas, 2008.

15. Brière N, Vallerand R, Blais N, Pelletier L. Développement et validation d'une mesure de motivation intrinsèque, extrinsèque et d'amotivation en contexte sportif : I'Échelle de motivation dans les sports (ÉMS). J of Sport Psychol 1995; 26(1): 465-489.

16. Barra Filho M, Andrade D, Miranda R, Núnez JL, Martin-Albó $\mathrm{J}$, Ribas PR. Preliminary validation of a brazilian version of the sport motivation scale. Univ Psychol 2011; 10(2): 557-566. DOI: 1657-9267(201108)10:2<557:PVOSMS>2.0.TX;2-Z.

17. Oslin JL, Mitchell SA, Griffin LL. The Game Performance Assessment Instrument (GPAI): development and preliminary validation. J. of Teach. in Phys. Educ 1998; 17(2): 231-243.

18. Memmert D, Harvey S. The Game Performance Assessment Instrument (GPAI): Some Concerns and Solutions for Further Development. J. of Teach. in Phys. Educ 2008; 27(1): 220-240.

19. Garganta J. Competências no ensino e treino de jovens futebolistas. Rev. Dig. Buenos Aires 2002; 45(8): 1-15.

20. Vieira LF, Mizoguchi MV, Garcia Junior E, Garcia WF. Estilos parentais e motivação em atletas jovens de futebol de campo. Pensar a prática 2013; 16(1): 183196. DOI: http://10.5216/ rpp.v16i1.16539.

21. Garcia-Mas A, Palou P, Gili M, Ponseti X, Borras PA, Vidal J, Cruz J, Torregrosa M, Villamarín F, Sousa C.. Commitment, Enjoyment and Motivation in Young Soccer Competitive Players. Span J Psychol 2010; 13(2): 609-616.

22. Balbinotti CAA, Barbosa MLL, Juchem L, Balbinotti MAA, Saldanha RP. A motivação à prática de atividade física regular relacionada ao prazer em adolescentes do sexo masculino. Coleção Pesquisa em Educação Física 2008; 7(1): 67-74. DOI: http://dx.doi.org/10.1590/S1807-55092008000100006.

23. Álvarez MS, Balaguer I, Castilho I, Duda JL. Coach Autonomy Support and Quality of Sport Engagement in Young Soccer Players. Span J Psychol 2009; 12(1): 138-148. 